\title{
Standardization of in vitro Culture Establishment and Proliferation of Micro-Shoots in African and French Marigold Genotypes
}

\author{
K. Ravindra Kumar ${ }^{1 *}$, Kanwar Pal Singh ${ }^{2}$, D.V.S. Raju ${ }^{3}$, Sapna Panwar ${ }^{2}$, \\ Reeta Bhatia ${ }^{4}$, Surendra Kumar ${ }^{2}$ and Pavanesh Kumar Verma ${ }^{2}$ \\ ${ }^{1}$ Dr.YSRHU, HRS-Kovvur, West Godavari Dist, Andhra Pradesh, India \\ ${ }^{2}$ ICAR-Indian Agricultural Research Institute, New Delhi, India \\ ${ }^{3}$ ICAR-Directorate of Floriculture Research, Pune, India \\ ${ }^{4}$ ICAR-IARI Regional Station, Katrain, Himachal Pradesh, India \\ *Corresponding author
}

\begin{tabular}{|l}
\hline Key w o r d s \\
African marigold, \\
French marigold, \\
Nodal segment, \\
Micropropagation, \\
Culture \\
establishment, \\
Vitrification, \\
Proliferation \\
\hline Article Info \\
\hline $\begin{array}{l}\text { Accepted: } \\
\text { 20 December } 2017 \\
\text { Available Online: } \\
\text { 10 January } 2018\end{array}$ \\
\hline
\end{tabular}

\section{Keywords}

African marigold,

Micropropagation,

Article Info

Accepted:

Available Online:
A B S T R A C T

Marigold is native to Mexico and one of the commercial loose flower crops in India. In general it is commonly propagated through seeds, but some ornamentally high valued petaloid and gynomonoecious lines can only be maintained through vegetative propagation. Initial in vitro axenic culture establishment, poor multiplication rates, excess callusing and vitrified cultures are the major hindrances in its commercial micro-propagation. Therefore, the objective of the present investigation was to develop efficient in vitro protocol for mass multiplication of commercially popular African and French marigold cultivars Pusa Basanti Gainda (PBG) and Pusa Arpita (PA) respectively. Nodal segments were chosen as explant of these two open field cultivars. Explants were pre-treated with carbendazim $(0.2 \%)+$ metalaxyl $(0.2 \%)+8$-hydroxy quinoline citrate $(200$ $\mathrm{mg} / \mathrm{l}$ ) for 60 minutes followed by surface sterilization with $0.1 \% \mathrm{HgCl}_{2}$ for 4 minutes to eliminate the microbial contamination. Highest culture establishment (69.44\%) and earliest bud emergence (4.45 days) was recorded in Murashige and Skoog (MS) medium supplemented with BAP $(2.0 \mathrm{mg} / \mathrm{l})$ and NAA $(0.05 \mathrm{mg} / \mathrm{l})$. Among the different proliferation treatments, $100 \%$ proliferation was recorded in MS medium devoid of any growth regulators, MS $+0.5 \mathrm{mg} / \mathrm{l}$ Kinetin $+0.1 \mathrm{mg} / \mathrm{l}$ $\mathrm{NAA}$ and $0.5 \mathrm{mg} / \mathrm{l} \mathrm{BAP}+0.1 \mathrm{mg} / \mathrm{l} \mathrm{NAA}+2.5 \mathrm{mg} / \mathrm{l} \mathrm{AgNO}_{3}$ supplemented media The maximum numbers of quality shoots $(4.3,18.8,64.2$ and 208.2 shoots/explant) were obtained on MS medium supplemented with $0.5 \mathrm{mg} / \mathrm{l} \mathrm{BAP}+$ $0.1 \mathrm{mg} / \mathrm{l} \mathrm{NAA}+2.5 \mathrm{mg} / 1 \mathrm{AgNO}_{3}$ in 30, 60, 90 and 120 days after culture respectively. This protocol is highly useful for mass multiplication of true-to-type, disease free planting material as well as helpful in long term maintenance of germplasm lines.

\section{Introduction}


Marigold is a member of the Asteraceae family and popular for commercial loose flower cultivation. It is a native of Mexico and naturalised in India about 350 years ago. Marigold is one of the high valued ornamental crop in India on account of its easy cultivation, short duration, vast adaptability, wide spectrum of shape, size and good keeping quality. Among the floriculture crops, it is cultivated in an area of 56.04 thousand ha. with 501.87 thousand MT production and occupied first in area and production (Anonymous, 2015). Apart from loose flower cultivation, it is also widely grown for extraction pigments (lutein) added to poultry feed for intensification yellow colour of egg yolk (Hojnik et al., 2008). It is also endowed with other properties like insecticide (pyrethrins), antibiotic, nematicide and fungicides (thiophenes). Marigold is sexually propagated through seeds. But, seed propagation has limited application in some of the popular petaloid commercial varieties, due to poor seed set, low viability and genetic segregation of progeny. These varieties are being propagated asexually through herbaceous shoot-tip cuttings for commercial cultivation. Tejaswini et al., (2016) reported the vegetative propagation of marigold petaloid and gynomonoecious lines in different breeding programmes. However, vegetative multiplication is cumbersome, slow, season dependent and one of the prime causes for spread of diseases like phyllody which is caused by phytoplasma. Plant tissue culture has the potential for rapid multiplication of a large number of diseasefree, true-to-type quality plants in the shortest possible time and can be employed as an alternative tool. Earlier, few workers demonstrated techniques of multiplication of marigold through shoot tip and axillary bud proliferation (Misra and Datta 2000, Kumar et al., 2003, Gupta et al., 2013 and Majumder et al., 2014). However, these results were not reproducible in nature.

Therefore, a study was conducted to develop an efficient and reproducible protocol for rapid in vitro propagation of commercially important African and French marigold cultivars.

\section{Materials and Methods}

The present experimentation was carried out at the Central Tissue Culture Laboratory, National Research Centre on Plant Biotechnology, New Delhi during 2014-2017. African marigold cv. Pusa Basanti Gainda (PBG) and French marigold cv. Pusa Arpita were used for the study (Fig. 1a \& b). In this research work, axillary shoots containing dormant buds were selected as explants. The explants were collected in early hours from the actively growing mother plants before the commencement of reproductive phase. The availability and quality of explants were observed to be low during flowering stage. Nodal segments of $2.0-2.5 \mathrm{~cm}$ length were excised and the leaf primordia removed with a sterile scalpel blade. Well prepared nodal segments were washed with Teepol ${ }^{\circledR}(0.1 \%)$ solution for 5 minutes followed by washing under running tap water for 10 minutes to remove the residue of the detergent. The explants were pre-treated with carbendazim $(0.2 \%)+$ metalaxyl $(0.2 \%)+8$-hydroxy quinoline citrate $(200 \mathrm{mg} / \mathrm{l})$ on a horizontal shaker (100 rpm) for 60 minutes followed by surface sterilization using $\mathrm{HgCl}_{2}(0.1 \%)$ for 4 minutes under laminar air-hood. The sterilised nodal segments were thoroughly washed with sterile double distilled water for 3 to 4 times to remove the chemical residues. The above treatments were used on the basis of initial experiments conducted by using different pretreatment and surface sterilisation combinations. The nodal segment was inoculated in each test tube $(150 \mathrm{~mm} \times 25$ $\mathrm{mm}$ ) with $15 \mathrm{ml}$ of modified Murashige and 
Skoog (MS) medium, supplemented with 3\% sucrose, $0.8 \%$ agar and various concentrations of BAP $(0-3.0 \mathrm{mg} / \mathrm{l})$ with NAA $(0.05 \mathrm{mg} / \mathrm{l})$ for culture initiation. Thereafter, the microshoots were excised from aseptic cultures and subculture at 30 days interval on proliferation media containing BAP (0 (T0), 0.5 (T1), 1.0 (T2), 1.5 (T3), 2.0 (T4) and 3.0 (T5) mg/l), kinetin (0.5 (T6) and 1.0 (T7) $\mathrm{mg} / \mathrm{l})$ individually and in combination $(0.5+0.5$ (T8), $1.0+0.5$ (T9) $\mathrm{mg} / \mathrm{l})$ with NAA $(0.1$ $\mathrm{mg} / \mathrm{l})$. On the basis of initial experiment results silver nitrate $(2.5 \mathrm{mg} / \mathrm{l})$ was tested with $0.5 \mathrm{mg} / \mathrm{l} \mathrm{BAP}$ and $0.1 \mathrm{mg} / \mathrm{l} \mathrm{NAA}$ as one of the proliferation treatment (T10). As $\mathrm{AgNO}_{3}$ is a thermolabile compound it was added to autoclaved medium after filter sterilisation with $0.22 \mu \mathrm{M}$ filters. To test the efficiency of different proliferation media and to determine the rate of proliferation the experiment was continued up to 120 days.

The cultures were maintained at $24 \pm 2{ }^{\circ} \mathrm{C}$ under fluorescent white light $\left(47 \mathrm{~mol} / \mathrm{m}^{2} / \mathrm{s}\right)$ at a photoperiod of 16/8 hours light and dark cycles. All cultures were examined periodically and observations on any morphological changes were recorded.

Twenty-five explants were inoculated per treatment and each treatment was replicated thrice and the reported data are mean of three replications. The data was statistically analysed employing completely randomised design. The percentage data were subjected to angular transformation before analysis.

\section{Results and Discussion}

\section{Pre-treatments}

Aseptic culture establishment is first and foremost step for the successful development of micro-propagation protocol on a commercial scale. In this study, various fungicides and bactericides were tried in different combinations and durations to eliminate the microbial contamination from the nodal explants. Among the different fungicidal treatments tried, explants agitation in carbendazim $(0.2 \%)+$ metalaxyl $(0.2 \%)+$ 8-hydroxy quinoline citrate $(200 \mathrm{mg} / \mathrm{l})$ for 60 minutes gave significantly higher survival $(66.67 \%)$ over other treatments (Table 1$)$. In comparison between the two genotypes, percent survival was significantly highest in Pusa Arpita $(32.06 \%)$ over Pusa Basanti Gainda (27.14\%). The two-way interaction between the pre-treatment and genotype was found to be non-significant. Under our experimental conditions, significantly lowest contamination $(26.67 \%)$ was observed in explant treated with carbendazim $(0.2 \%)+$ metalaxyl $(0.2 \%)+8$-hydroxy quinoline citrate $(200 \mathrm{mg} / \mathrm{l})$ for 90 minutes, which was followed by 60 minutes duration (30.00) of treatment. However, the survival percentage $(8.89 \%)$ was significantly low when explants were treated for 90 minutes. This might be due to the toxic effect of chemicals under prolonged duration of treatment (Table 1). All pre-treatments gave significantly better response compared to control, where 98.33 percent contamination was noted. Microbes such as bacteria and fungi were responsible for culture contamination and can completely spoil the cultures. Among the different pretreatments, highest explant toxicity $(64.44 \%)$ was recorded with highest fungicide dosage and prolonged (90 min) treatment duration (Table 1). These findings are in close confirmation with earlier results reported by Singh et al., (2011) in grape, Verma et al., (2012) in chrysanthemum and Sen et al., (2013) in Achyranthes aspera L. Most of these findings proved the usefulness of carbendazim $(0.1-3.0 \%)$ and metalaxyl $(0.1-3.0 \%)$ as effective fungicides. Fungicide dosage and treatment duration depend on the type and tenderness of explant. But higher concentrations of these disinfectants and prolonged durations of treatment became toxic 
and were responsible for poor growth and low establishment of cultures particularly in herbaceous crops.

\section{Surface sterilization}

Standardisation of surface sterilisation treatment followed by efficient pre-treatment is a vital process for axenic culture establishment.

It is clear from the Table 2 that significantly higher survival $(73.3 \%)$ was recorded when the explants were pre-treated with carbendazim $(0.2 \%)+$ metalaxyl $(0.2 \%)+8$ hydroxy quinoline citrate $(200 \mathrm{mg} / \mathrm{l})$ for 60 minutes followed by 4 minutes $\mathrm{HgCl}_{2}(0.1 \%)$ treatment over all other treatments. It was also observed that explants were killed when treatment duration was increased beyond 4 minutes in $\mathrm{HgCl}_{2}(0.1 \%)$. This might be due to the toxic effect of surface sterilant on explants (Table 2). It was clearly evident from the data, $\mathrm{NaOCl}(4 \%)$ treatment for 15 and 20 minutes was less efficient than $\mathrm{HgCl}_{2}(0.1 \%)$ for 4 minutes in controlling the microbial contamination.

Among the two genotypes, per cent survival was significantly highest in Pusa Arpita (41.70\%) over Pusa Basanti Gainda (37.20\%). The two-way interaction between the surface sterilant and genotype was found to be nonsignificant. Our research finding revealed that explants treated with $\mathrm{HgCl}_{2}(0.1 \%)$ for short duration ( $<3$ minutes) failed to kill the microbes effectively, whereas longer durations (5 to 8 minutes) resulted in complete or partial tissue killing in both the species of marigold. Treating the explants with $\mathrm{HgCl}_{2}(0.1 \%)$ for 4 minutes resulted in higher survival of explants with low contamination $(24.4 \%)$. Our results are in tantamount to Singh et al., (2011) in grape and Verma et al., (2012) in chrysanthemum. But these results are in contrary with Majumder et al., (2014), where they reported only 2 minutes treatment with $\mathrm{HgCl}_{2}(0.1 \%)$ resulted in highest culture establishment in Pusa Narangi Gainda and the variation might be due to change in the genotype.

\section{Culture initiation}

Different BAP concentrations $(0,0.5,1.0,2.0$ and $3.0 \mathrm{mg} / \mathrm{l}$ ) were tried along with NAA $(0.05 \mathrm{mg} / \mathrm{l})$ for culture establishment (Table $3)$. Under our experimental conditions, among the different growth regulators tested, the highest culture establishment $(69.44 \%)$ was noted with $2.0 \mathrm{mg} / \mathrm{l} \mathrm{BAP}+0.05 \mathrm{mg} / \mathrm{l} \mathrm{NAA}$, followed by $1.0 \mathrm{mg} / \mathrm{l} \mathrm{BAP}+0.05 \mathrm{mg} / \mathrm{l} \mathrm{NAA}$ $(56.11 \%)$, which were significantly different (Fig 2 a \& b). The culture establishment was higher in the genotype Pusa Arpita (49.33\%) followed by Pusa Basanti Gainda (46.89\%) both are at par with each other. The interaction between treatment and genotype was also insignificant.

Early (4.45 days) bud sprouting was observed on MS medium supplemented with $2.0 \mathrm{mg} / \mathrm{l}$ $\mathrm{BAP}+0.05 \mathrm{mg} / \mathrm{l} \mathrm{NAA}$, followed by $3.0 \mathrm{mg} / \mathrm{l}$ $\mathrm{BAP}+0.05 \mathrm{mg} / \mathrm{l} \mathrm{NAA}$ (4.82 days), which were statistically significant with each other. Explants cultured on MS medium devoid of any growth regulators took longer duration (11.55 days) for axillary bud sprouting. Among the genotypes, significantly earlier axillary bud sprouting (6.77 days) was recorded in Pusa Arpita compared to Pusa Basanti Gainda (7.55 days). The interaction between growth regulator and genotype was also found significant.

Duration for bud sprouting was the earlier in Pusa Arpita (4.07 days) than Pusa Basanti Gainda (4.83 days) when they were cultured on MS medium supplemented with $2.0 \mathrm{mg} / \mathrm{l}$ $\mathrm{BAP}+0.05 \mathrm{mg} / \mathrm{l} \mathrm{NAA}$ treatment (Table 3). 
Table.1 Effect of different pre-treatments in the sterilization of nodal explants in African marigold cv. Pusa Basanti Gainda (PBG) and French marigold cv. Pusa Arpita (PA)

\begin{tabular}{|c|c|c|c|c|c|c|c|c|c|c|c|}
\hline \multirow[t]{2}{*}{ Treatment } & \multirow[t]{2}{*}{ Treatment details } & \multirow{2}{*}{$\begin{array}{l}\text { Duration } \\
\text { (minutes) }\end{array}$} & \multicolumn{2}{|c|}{ Survival $(\%)$} & \multirow[t]{2}{*}{ Mean } & \multicolumn{2}{|c|}{ Contamination $(\%)$} & \multirow[t]{2}{*}{ Mean } & \multicolumn{2}{|c|}{ Toxicity $(\%)$} & \multirow[t]{2}{*}{ Mean } \\
\hline & & & PBG & PA & & PBG & PA & & PBG & PA & \\
\hline T0 & Control (Distilled water shake) & 60 & $\begin{array}{c}1.11 \\
(3.50)^{*}\end{array}$ & $\begin{array}{c}2.22 \\
(7.01)^{*}\end{array}$ & 1.67 & $\begin{array}{c}98.89 \\
(85.25)^{*}\end{array}$ & $\begin{array}{c}97.78 \\
(82.35)^{*}\end{array}$ & 98.33 & $\begin{array}{c}0.00 \\
(0.00)^{*}\end{array}$ & $\begin{array}{c}0.00 \\
(0.00)^{*}\end{array}$ & 0.00 \\
\hline T1 & $\begin{array}{l}\text { Carbendazim }(0.1 \%)+\text { Metalaxyl } \\
(0.1 \%)+8 \text {-HQC }(200 \mathrm{mg} / \mathrm{l})\end{array}$ & 30 & $\begin{array}{c}27.78 \\
(31.79)\end{array}$ & $\begin{array}{c}31.11 \\
(33.88)\end{array}$ & 29.44 & $\begin{array}{c}71.11 \\
(57.49)\end{array}$ & $\begin{array}{c}66.67 \\
(54.73)\end{array}$ & 68.89 & $\begin{array}{c}1.11 \\
(3.50)\end{array}$ & $\begin{array}{c}2.22 \\
(7.01)\end{array}$ & 1.67 \\
\hline $\mathbf{T 2}$ & $\begin{array}{l}\text { Carbendazim }(0.1 \%)+\text { Metalaxyl } \\
(0.1 \%)+8-H Q C(200 \mathrm{mg} / \mathrm{l})\end{array}$ & 60 & $\begin{array}{c}37.78 \\
(37.88)\end{array}$ & $\begin{array}{c}46.67 \\
(43.06)\end{array}$ & 42.22 & $\begin{array}{c}60.00 \\
(50.77)\end{array}$ & $\begin{array}{c}50.00 \\
(44.99)\end{array}$ & 55.00 & $\begin{array}{c}2.22 \\
(7.01)\end{array}$ & $\begin{array}{c}3.33 \\
(8.49)\end{array}$ & 2.78 \\
\hline T3 & $\begin{array}{l}\text { Carbendazim }(0.1 \%)+\text { Metalaxyl } \\
(0.1 \%)+8-\mathrm{HQC}(200 \mathrm{mg} / \mathrm{l})\end{array}$ & 90 & $\begin{array}{c}15.56 \\
(23.02)\end{array}$ & $\begin{array}{l}22.22 \\
(28.01)\end{array}$ & 18.89 & $\begin{array}{c}41.11 \\
(39.82)\end{array}$ & $\begin{array}{c}30.00 \\
(33.18)\end{array}$ & 35.56 & $\begin{array}{c}43.33 \\
(41.09)\end{array}$ & $\begin{array}{c}47.78 \\
(43.69)\end{array}$ & 45.56 \\
\hline T4 & $\begin{array}{l}\text { Carbendazim }(0.2 \%)+\text { Metalaxyl } \\
(0.2 \%)+8 \text {-HQC }(200 \mathrm{mg} / \mathrm{l})\end{array}$ & 30 & $\begin{array}{c}38.89 \\
(38.55)\end{array}$ & $\begin{array}{c}40.00 \\
(39.20)\end{array}$ & 39.44 & $\begin{array}{c}57.78 \\
(49.48)\end{array}$ & $\begin{array}{c}56.67 \\
(48.82)\end{array}$ & 57.22 & $\begin{array}{c}3.33 \\
(8.49)\end{array}$ & $\begin{array}{c}3.33 \\
(8.49)\end{array}$ & 3.33 \\
\hline T5 & $\begin{array}{l}\text { Carbendazim }(0.2 \%)+\text { Metalaxyl } \\
(0.2 \%)+8 \text {-HQC }(200 \mathrm{mg} / \mathrm{l})\end{array}$ & 60 & $\begin{array}{c}61.11 \\
(51.44)\end{array}$ & $\begin{array}{c}72.22 \\
(58.33)\end{array}$ & 66.67 & $\begin{array}{c}34.44 \\
(35.89)\end{array}$ & $\begin{array}{c}25.56 \\
(30.28)\end{array}$ & 30.00 & $\begin{array}{c}4.44 \\
(11.99)\end{array}$ & $\begin{array}{c}2.22 \\
(14.96)\end{array}$ & 3.33 \\
\hline \multirow[t]{6}{*}{ T6 } & $\begin{array}{l}\text { Carbendazim }(0.2 \%)+\text { Metalaxyl } \\
(0.2 \%)+8 \text {-HQC }(200 \mathrm{mg} / \mathrm{l})\end{array}$ & 90 & $\begin{array}{c}7.78 \\
(15.63)\end{array}$ & $\begin{array}{c}10.00 \\
(18.00)\end{array}$ & 8.89 & $\begin{array}{c}31.11 \\
(33.84)\end{array}$ & $\begin{array}{c}22.22 \\
(28.01)\end{array}$ & 26.67 & $\begin{array}{c}61.11 \\
(51.43)\end{array}$ & $\begin{array}{c}67.78 \\
(55.55)\end{array}$ & 64.44 \\
\hline & Mean & & 27.14 & 32.06 & & 56.35 & 49.84 & & 16.51 & 18.10 & \\
\hline & & & $\begin{array}{c}C D \\
(p<0.05)\end{array}$ & SEm \pm & & $\begin{array}{c}C D \\
(p<0.05)\end{array}$ & SEm \pm & & $\begin{array}{c}\mathrm{CD} \\
(\mathrm{p}<0.05)\end{array}$ & SEm \pm & \\
\hline & Treatments & & 4.88 & 1.69 & & 6.37 & 2.20 & & 6.37 & 2.20 & \\
\hline & Genotype & & 2.61 & 0.9012 & & NS & 1.18 & & NS & 1.18 & \\
\hline & $\mathrm{T} \times \mathrm{G}$ & & NS & 2.38 & & NS & 3.11 & & NS & 3.11 & \\
\hline
\end{tabular}

*Figures given in parentheses are angular transformed values 
Table.2 Effect of different surface sterilisation treatments of nodal explants in African marigold cv. Pusa Basanti Gainda (PBG) and French marigold cv. Pusa Arpita (PA)

\begin{tabular}{|c|c|c|c|c|c|c|c|c|c|c|}
\hline \multirow[t]{2}{*}{ Treatment } & \multirow[t]{2}{*}{ Treatment details } & \multicolumn{2}{|c|}{ Survival (\%) } & \multirow[t]{2}{*}{ Mean } & \multicolumn{2}{|c|}{ Contamination $(\%)$} & \multirow[t]{2}{*}{ Mean } & \multicolumn{2}{|c|}{ Toxicity (\%) } & \multirow[t]{2}{*}{ Mean } \\
\hline & & PBG & PA & & PBG & PA & & PBG & PA & \\
\hline T0 & Control (Distilled water shake) & $\begin{array}{c}2.2 \\
(7.0)^{*}\end{array}$ & $\begin{array}{c}3.3 \\
(8.5)^{*}\end{array}$ & 2.8 & $97.8(82.8)^{*}$ & $\begin{array}{c}96.7 \\
(81.3)^{*}\end{array}$ & 97.2 & $0.0(0.00)^{*}$ & $\begin{array}{c}0.0 \\
(0.00)^{*}\end{array}$ & 0.0 \\
\hline T1 & $0.1 \% \mathrm{HgCl}_{2}$ for $3 \mathrm{~min}$. & $60.0(50.8)$ & $\begin{array}{c}68.9 \\
(56.1)\end{array}$ & 64.4 & $40.0(39.2)$ & $\begin{array}{c}31.1 \\
(33.9)\end{array}$ & 35.6 & $0.0(0.00)$ & $\begin{array}{c}0.0 \\
(0.00)\end{array}$ & 0.0 \\
\hline $\mathbf{T} 2$ & $0.1 \% \mathrm{HgCl}_{2}$ for $4 \mathrm{~min}$. & $71.1(57.6)$ & $\begin{array}{c}75.6 \\
(60.4)\end{array}$ & 73.3 & $27.8(31.7)$ & $\begin{array}{c}21.1 \\
(27.3)\end{array}$ & 24.4 & $\begin{array}{c}1.1 \\
(3.5)\end{array}$ & $\begin{array}{c}3.3 \\
(8.5)\end{array}$ & 2.2 \\
\hline T3 & $0.1 \% \mathrm{HgCl}_{2}$ for $5 \mathrm{~min}$. & $52.2(46.3)$ & $\begin{array}{c}58.9 \\
(50.1)\end{array}$ & 55.6 & $22.2(28.0)$ & $\begin{array}{c}17.8 \\
(24.9)\end{array}$ & 20.0 & $25.6(30.1)$ & $\begin{array}{c}23.3 \\
(28.6)\end{array}$ & 24.4 \\
\hline T4 & $0.1 \% \mathrm{HgCl}_{2}$ for $6 \mathrm{~min}$. & $23.3(28.8)$ & $\begin{array}{c}27.8 \\
(31.8)\end{array}$ & 25.6 & $17.8(24.9)$ & $\begin{array}{c}17.8 \\
(24.8)\end{array}$ & 17.8 & $58.9(50.1)$ & $\begin{array}{c}54.4 \\
(47.5)\end{array}$ & 56.7 \\
\hline T5 & $0.1 \% \mathrm{HgCl}_{2}$ for $7 \mathrm{~min}$. & $\begin{array}{c}8.9 \\
(17.1)\end{array}$ & $\begin{array}{c}17.8 \\
(24.9)\end{array}$ & 13.3 & $14.4(22.3)$ & $\begin{array}{c}3.3 \\
(8.5)\end{array}$ & 8.9 & $76.7(61.1)$ & $\begin{array}{c}78.9 \\
(62.7)\end{array}$ & 77.8 \\
\hline T6 & $0.1 \% \mathrm{HgCl}_{2}$ for $8 \mathrm{~min}$. & $\begin{array}{c}3.3 \\
(8.5)\end{array}$ & $\begin{array}{c}5.6 \\
(13.1)\end{array}$ & 4.4 & $5.6(13.5)$ & $\begin{array}{c}1.1 \\
(3.5)\end{array}$ & 3.3 & $91.1(73.2)$ & $\begin{array}{c}93.3 \\
(75.8)\end{array}$ & 92.2 \\
\hline T7 & $4.0 \% \mathrm{NaOCl}$ for $15 \mathrm{~min}$. & $51.1(45.6)$ & $\begin{array}{c}52.2 \\
(46.3)\end{array}$ & 51.7 & $46.7(43.0)$ & $\begin{array}{c}44.4 \\
(41.8)\end{array}$ & 45.6 & $\begin{array}{c}2.2 \\
(7.0)\end{array}$ & $\begin{array}{c}3.3 \\
(8.5)\end{array}$ & 2.8 \\
\hline \multirow[t]{6}{*}{ T8 } & $4.0 \% \mathrm{NaOCl}$ for $20 \mathrm{~min}$. & $62.2(52.1)$ & $\begin{array}{c}65.6 \\
(54.1)\end{array}$ & 63.9 & $32.2(34.6)$ & $\begin{array}{c}27.8 \\
(31.8)\end{array}$ & 30.0 & $5.6(13.1)$ & $\begin{array}{c}6.7 \\
(14.6)\end{array}$ & 6.1 \\
\hline & Mean & 37.2 & 41.7 & & 33.8 & 29.0 & & 29.0 & 29.3 & \\
\hline & & $\mathrm{CD}(\mathrm{p}<0.05)$ & SEm \pm & & $\mathrm{CD}(\mathrm{p}<0.05)$ & SEm \pm & & $\mathrm{CD}(\mathrm{p}<0.05)$ & SEm \pm & \\
\hline & Treatments & 5.33 & 1.86 & & 4.87 & 1.69 & & 5.58 & 1.95 & \\
\hline & Genotype & 2.51 & 0.90 & & 2.29 & 0.80 & & NS & 0.90 & \\
\hline & $\mathrm{T} \times \mathrm{G}$ & NS & 2.60 & & NS & 2.4 & & NS & 2.80 & \\
\hline
\end{tabular}

\footnotetext{
*Figures given in parentheses are angular transformed values
} 
Table.3 Effect of BAP and NAA on in vitro culture establishment (\%), days to bud sprouting, no. of shoots per explants, avg. shoot length $(\mathrm{cm})$ and callusing after 25 days after culture initiation in African marigold cv. Pusa Basanti Gainda (PBG) and French marigold cv. Pusa Arpita (PA)

\begin{tabular}{|c|c|c|c|c|c|c|c|c|c|c|c|c|c|c|c|c|c|}
\hline \multirow[t]{2}{*}{$\begin{array}{l}\text { Treat } \\
\text { ment }\end{array}$} & \multicolumn{2}{|c|}{$\begin{array}{l}\text { Growth } \\
\text { regulators } \\
(\mathrm{mg} / \mathrm{l})\end{array}$} & \multicolumn{2}{|c|}{$\begin{array}{c}\text { Culture } \\
\text { establishment } \\
(\%)\end{array}$} & \multirow[t]{2}{*}{ Mean } & \multicolumn{2}{|c|}{$\begin{array}{l}\text { Days to bud } \\
\text { sprouting }\end{array}$} & \multirow[t]{2}{*}{ Mean } & \multicolumn{2}{|c|}{$\begin{array}{c}\text { Shoots per } \\
\text { explant }\end{array}$} & \multirow[t]{2}{*}{ Mean } & \multicolumn{2}{|c|}{$\begin{array}{l}\text { Av. shoot } \\
\text { length }(\mathrm{cm})\end{array}$} & \multirow[t]{2}{*}{ Mean } & \multicolumn{2}{|c|}{$\begin{array}{l}\text { Establishment } \\
\text { Index }\end{array}$} & \multirow[t]{2}{*}{ Mean } \\
\hline & $\begin{array}{c}\mathrm{BA} \\
\mathrm{P}\end{array}$ & $\begin{array}{c}\text { NA } \\
\text { A }\end{array}$ & PBG & PA & & PBG & PA & & PBG & $\mathbf{P A}$ & & PBG & PA & & PBG & PA & \\
\hline T0 & 0.0 & 0.00 & $\begin{array}{c}30.00 \\
(33.18 \\
)^{*}\end{array}$ & $\begin{array}{c}27.78 \\
(31.73 \\
)^{*}\end{array}$ & 28.89 & 12.13 & 10.97 & 11.55 & 1.00 & 1.00 & 1.00 & 0.73 & 0.67 & 0.70 & 30.0 & 27.70 & 28.8 \\
\hline T1 & 0.5 & 0.05 & $\begin{array}{c}42.22 \\
(40.46 \\
)\end{array}$ & $\begin{array}{c}46.67 \\
(43.06 \\
)\end{array}$ & 44.44 & 8.30 & 7.30 & 7.80 & 1.00 & 1.00 & 1.00 & 1.13 & 0.88 & 1.01 & 42.2 & 46.60 & 44.4 \\
\hline $\mathbf{T} 2$ & 1.0 & 0.05 & $\begin{array}{c}52.22 \\
(46.27 \\
)\end{array}$ & $\begin{array}{c}60.00 \\
(50.75 \\
)\end{array}$ & 56.11 & 7.56 & 6.80 & 7.18 & 1.55 & 1.30 & 1.43 & 1.47 & 1.40 & 1.43 & 80.8 & 77.60 & 79.2 \\
\hline T3 & 2.0 & 0.05 & $\begin{array}{c}72.22 \\
(58.30 \\
)\end{array}$ & $\begin{array}{c}66.67 \\
(54.78 \\
)\end{array}$ & 69.44 & 4.83 & 4.07 & 4.45 & 1.92 & 1.83 & 1.88 & 2.17 & 2.00 & 2.08 & 138.0 & 122.3 & 130.2 \\
\hline T4 & 3.0 & 0.05 & $\begin{array}{c}37.78 \\
(37.90 \\
)\end{array}$ & $\begin{array}{c}45.56 \\
(42.42 \\
)\end{array}$ & 41.67 & 4.90 & 4.73 & 4.82 & 1.63 & 1.54 & 1.58 & 1.63 & 1.57 & 1.60 & 62.0 & 69.90 & 66.0 \\
\hline \multirow{2}{*}{\multicolumn{3}{|c|}{ Mean }} & 46.89 & 49.33 & & 7.55 & 6.77 & & 1.42 & 1.33 & & 1.43 & 1.30 & & 70.6 & 68.88 & \\
\hline & & & $\begin{array}{c}C D \\
(p<0.0 \\
5)\end{array}$ & SEm \pm & & $\begin{array}{c}C D \\
(p<0.05 \\
)\end{array}$ & $\begin{array}{l}\text { SEm } \\
\pm\end{array}$ & & $\begin{array}{c}C D \\
(p<0.05 \\
)\end{array}$ & SEm \pm & & $\begin{array}{c}C D \\
(p<0.0 \\
5)\end{array}$ & $\begin{array}{l}\mathrm{SE} \\
\mathrm{m} \pm\end{array}$ & & $\begin{array}{c}C D \\
(p<0.05 \\
)\end{array}$ & SEm \pm & \\
\hline \multicolumn{3}{|c|}{ Treatments } & 4.28 & 1.441 & & 0.857 & 0.29 & & 0.12 & 0.04 & & 0.22 & 0.08 & & 10.54 & 3.57 & \\
\hline \multicolumn{3}{|c|}{ Genotype } & NS & 0.911 & & 0.542 & 0.18 & & 0.077 & 0.026 & & NS & 0.05 & & NS & 2.25 & \\
\hline \multicolumn{3}{|c|}{ T X G } & NS & 2.038 & & NS & 0.41 & & NS & 0.058 & & NS & 0.11 & & NS & 5.05 & \\
\hline
\end{tabular}

*Figures given in parentheses are angular transformed values 
Table.4 Effect of BAP, Kinetin, NAA and $\mathrm{AgNO}_{3}$ on micro-shoot proliferation in African marigold cv. Pusa Basanti Gainda (PBG) and French marigold cv. Pusa Arpita (PA)

\begin{tabular}{|c|c|c|c|c|c|c|c|c|c|c|}
\hline \multirow{3}{*}{$\begin{array}{l}\text { Treat } \\
\text { ment }\end{array}$} & \multirow{2}{*}{\multicolumn{4}{|c|}{ Treatment details (mg/l) }} & \multicolumn{2}{|c|}{ Proliferation (\%) } & \multirow{3}{*}{ Mean } & \multicolumn{2}{|c|}{ Average shoot length } & \multirow[t]{3}{*}{ Mean } \\
\hline & & & & & \multirow[t]{2}{*}{ PBG } & \multirow[t]{2}{*}{ PA } & & \multirow[t]{2}{*}{ PBG } & \multirow[t]{2}{*}{ PA } & \\
\hline & BAP & Kinetin & NAA & $\mathrm{AgNO}_{3}$ & & & & & & \\
\hline T0 & 0 & 0 & 0 & 0 & 100.0 & 100.0 & 100.0 & 3.7 & 4.5 & 4.1 \\
\hline T1 & 0.5 & 0 & 0.1 & 0 & 100.0 & 96.7 & 98.3 & 2.3 & 2.0 & 2.2 \\
\hline T2 & 1.0 & 0 & 0.1 & 0 & 96.7 & 83.3 & 90.0 & 0.6 & 0.9 & 0.7 \\
\hline T3 & 1.5 & 0 & 0.1 & 0 & 70.0 & 56.7 & 63.3 & 0.0 & 0.0 & 0.0 \\
\hline T4 & 2.0 & 0 & 0.1 & 0 & 36.7 & 30.0 & 33.3 & 0.0 & 0.0 & 0.0 \\
\hline T5 & 3.0 & 0 & 0.1 & 0 & 23.3 & 16.7 & 20.0 & 0.0 & 0.0 & 0.0 \\
\hline T6 & 0 & 0.5 & 0.1 & 0 & 100.0 & 100.0 & 100.0 & 1.5 & 2.0 & 1.7 \\
\hline T7 & 0 & 1.0 & 0.1 & 0 & 63.3 & 56.7 & 60.0 & 0.8 & 1.0 & 0.9 \\
\hline T8 & 0.5 & 0.5 & 0.1 & 0 & 43.3 & 33.3 & 38.3 & 0.4 & 0.8 & 0.6 \\
\hline T9 & 1.0 & 0.5 & 0.1 & 0 & 23.3 & 13.3 & 18.3 & 0.0 & 0.0 & 0.0 \\
\hline \multirow[t]{6}{*}{ T10 } & 0.5 & 0 & 0.1 & 2.5 & 100.0 & 100.0 & 100.0 & 2.8 & 2.6 & 2.7 \\
\hline & \multirow{2}{*}{\multicolumn{4}{|c|}{ Mean }} & 68.8 & 62.4 & & 1.1 & 1.2 & \\
\hline & & & & & $\mathrm{CD}(\mathrm{p}<0.05)$ & SEm \pm & & $\mathrm{CD}(\mathrm{p}<0.05)$ & $\mathrm{SEm} \pm$ & \\
\hline & \multicolumn{4}{|c|}{ Treatments } & 5.870 & 2.060 & & 0.508 & 0.178 & \\
\hline & \multicolumn{4}{|c|}{ Genotype } & 2.500 & 0.878 & & NS & 0.076 & \\
\hline & \multicolumn{4}{|c|}{$\mathrm{T} X \mathrm{XG}$} & NS & 2.910 & & NS & 0.251 & \\
\hline
\end{tabular}

*Figures given in parentheses are angular transformed values 
Table. 5 Effect of BAP, Kinetin, NAA and $\mathrm{AgNO}_{3}$ on number of micro-shoots per explant after 30, 60, 90 and 120 days of proliferation in African marigold cv. Pusa Basanti Gainda (PBG) and French marigold cv. Pusa Arpita (PA)

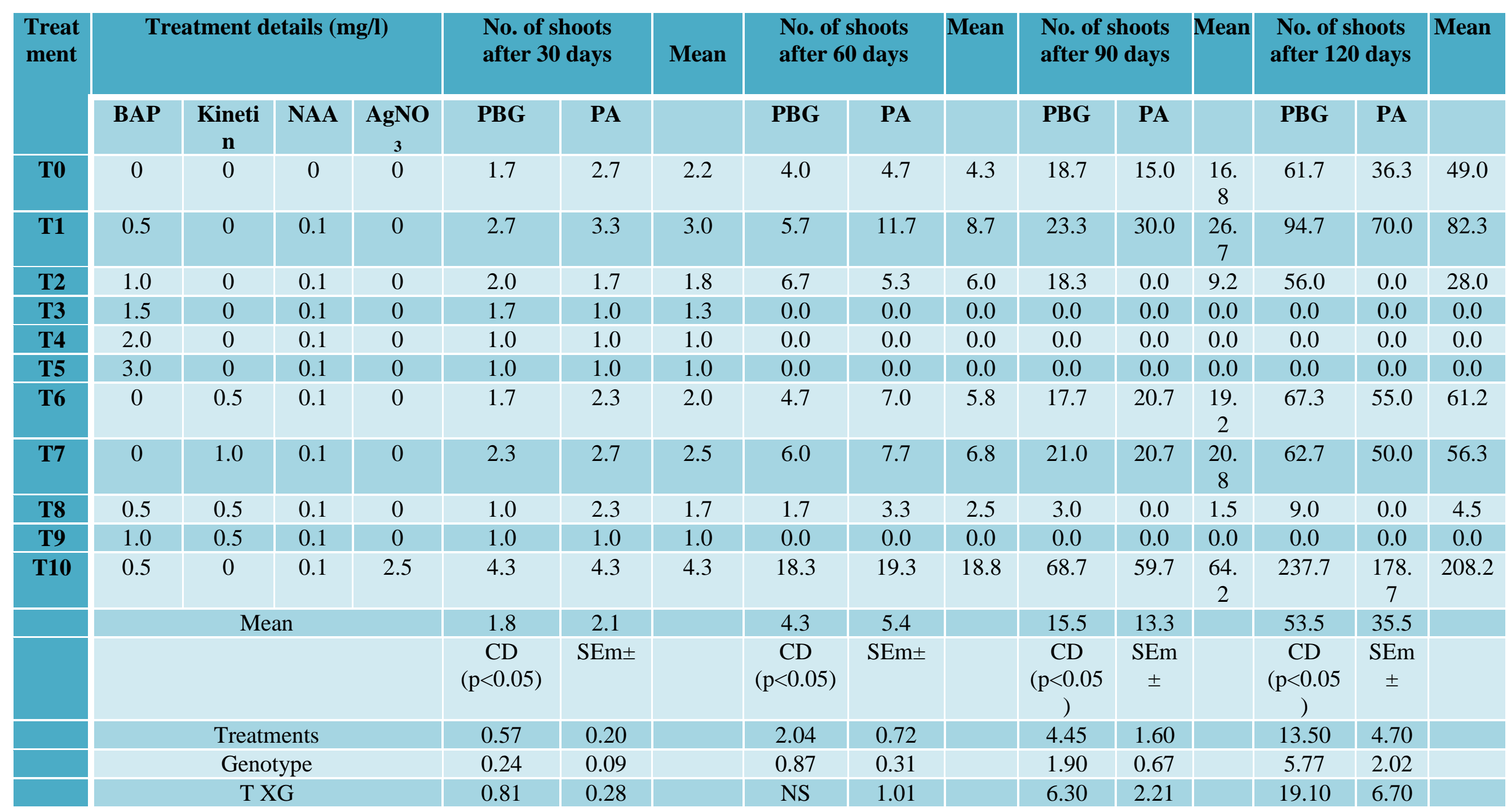



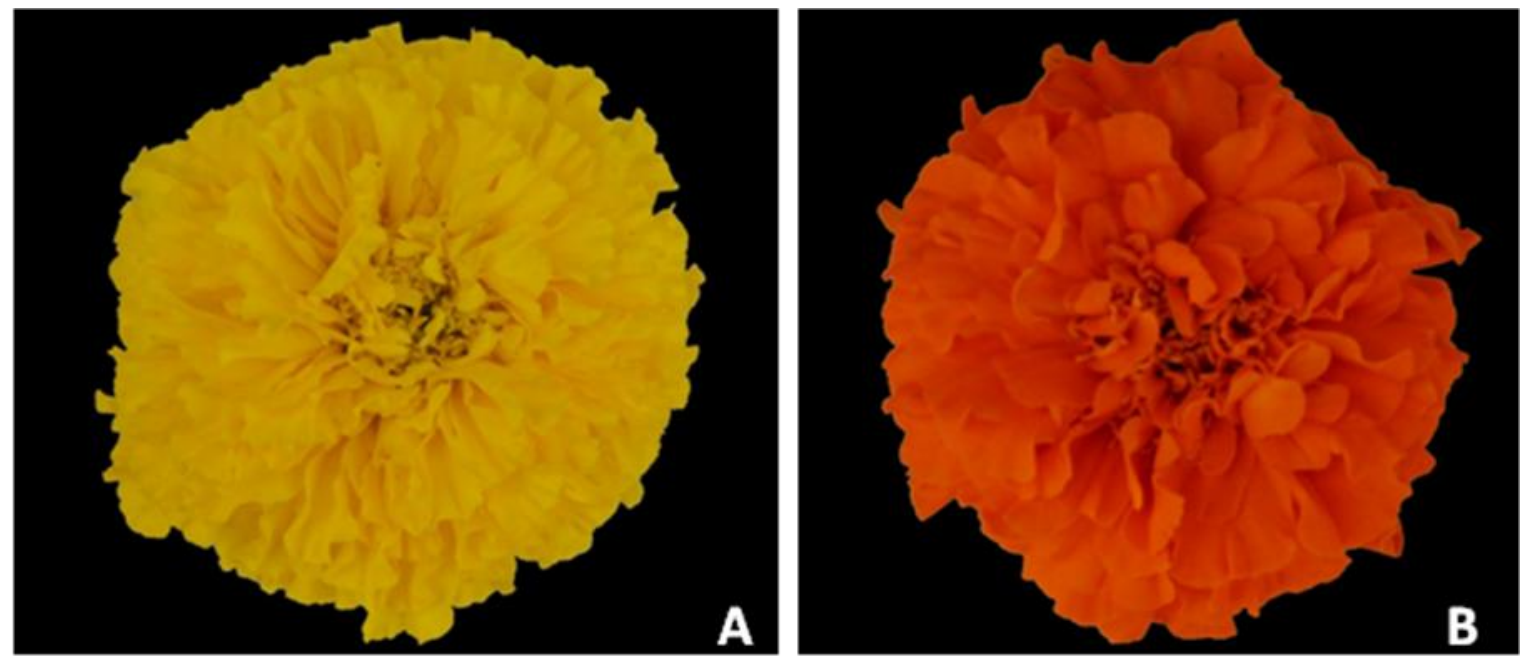

Fig. 1 A \& B Flowers of Pusa Basanti Gainda and Pusa Arpita respectively
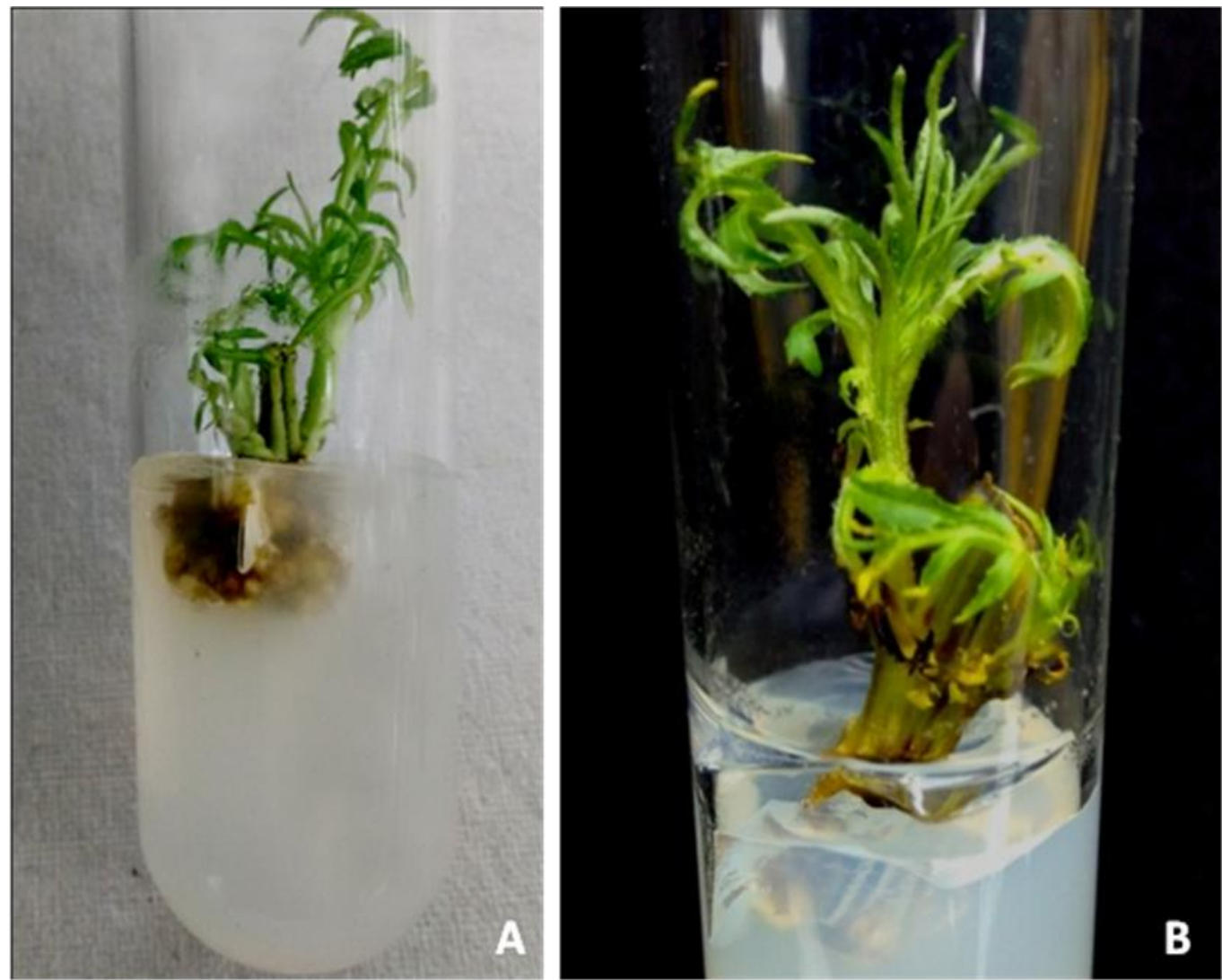

Fig. 2 Axillary bud sprouting on MS medium supplemented with $2.0 \mathrm{mg} / \mathrm{l}$ BAP + $0.05 \mathrm{mg} / \mathrm{I}$ NAA A) Pusa Basanti Gainda; B) Pusa Arpita 

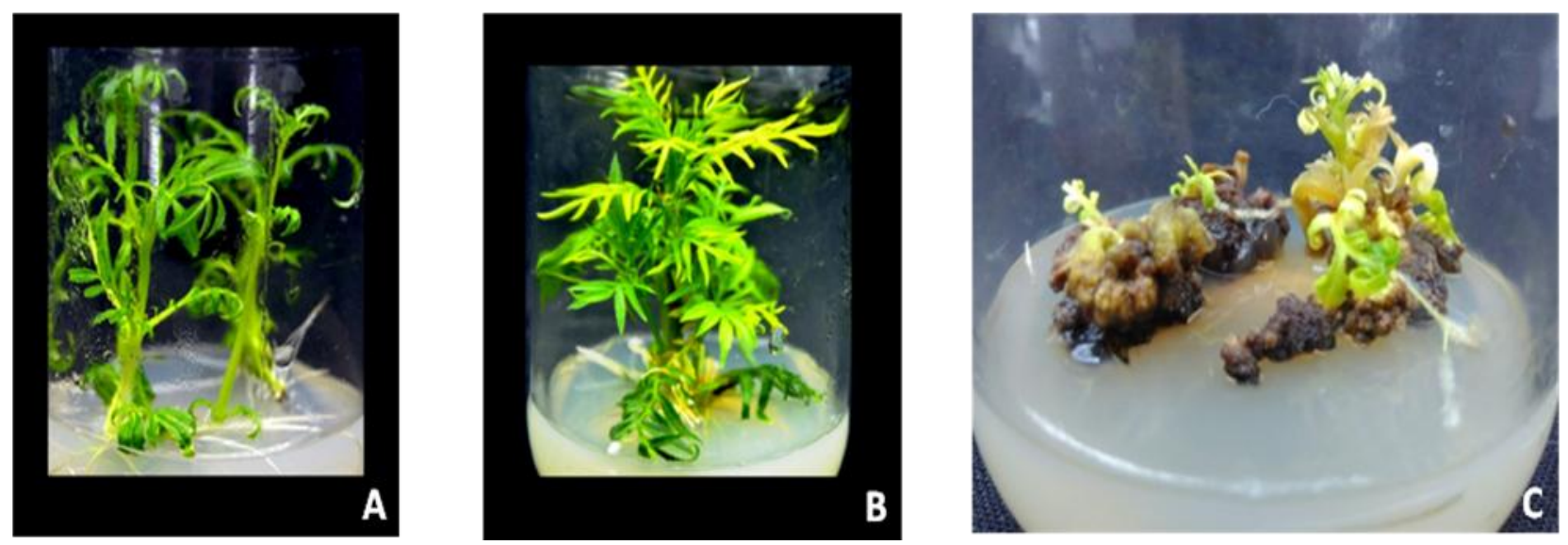

Fig .3 Shoot proliferation in African marigold cv. Pusa Basanti Gainda A) Proliferation of shoots on MS media devoid of growth regulators (Control) ; B) Proliferation on $\mathrm{MS}+0.5+0.1+2.5 \mathrm{mg} / \mathrm{BAP}, \mathrm{NAA}$ and $\left.\mathrm{AgNO}_{3} ; \mathrm{C}\right)$ Callus growth and vitrified shoots on MS + $\mathrm{MS}+0.5+0.5+0.1+2.5 \mathrm{mg} / \mathrm{l} \mathrm{BAP}$, Kinetin and NAA

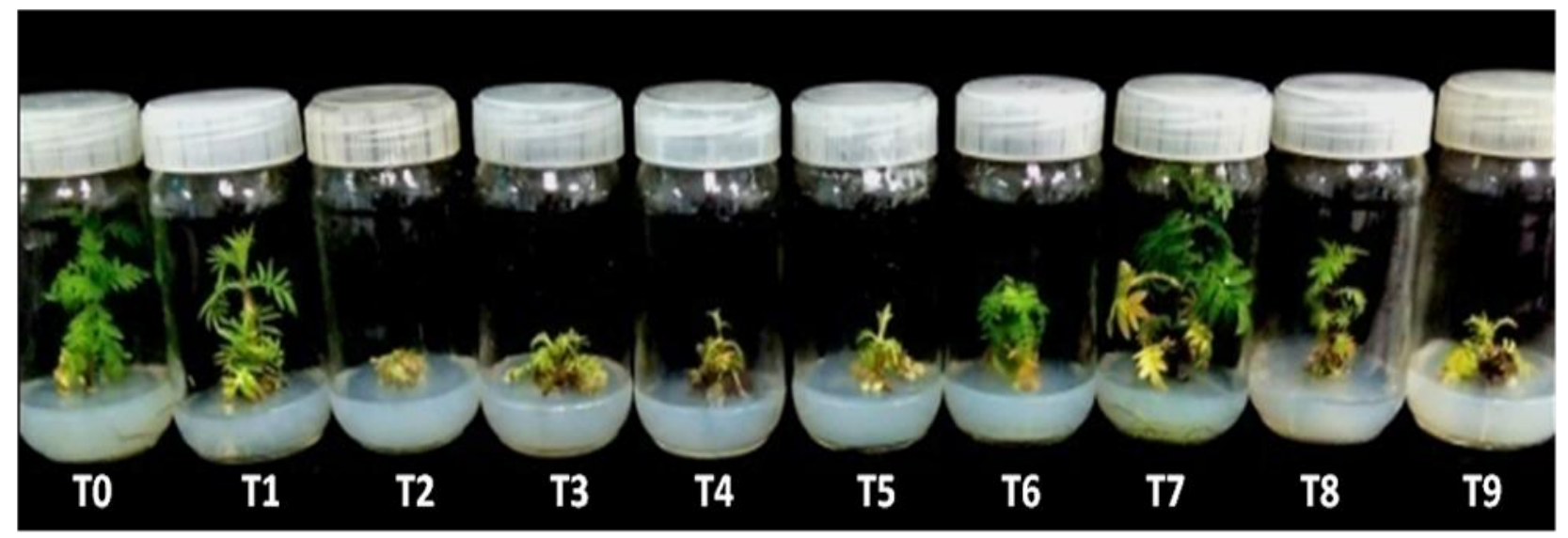

Fig .4 Shoot proliferation in French marigold cv. Pusa Arpita Proliferation of shoots in different treatments

Perusal of data from Table 3 revealed that, maximum number of micro-shoots per explant (1.88) was recorded in $2.0 \mathrm{mg} / \mathrm{l} \mathrm{BAP}$ $+0.05 \mathrm{mg} / \mathrm{l} \mathrm{NAA}$, which was statistically superior with all other treatments. The genotype response to different BAP concentrations and the interaction between treatment and genotype was insignificant in terms of number of micro-shoots per explants. Among the treatments significantly longest micro-shoots $(2.08 \mathrm{~cm})$ were obtained with
$2.0 \mathrm{mg} / \mathrm{l} \mathrm{BAP}+0.05 \mathrm{mg} / \mathrm{l} \mathrm{NAA}$ treatment followed by $3.0 \mathrm{mg} / \mathrm{l} \mathrm{BAP}+0.05 \mathrm{mg} / \mathrm{l} \mathrm{NAA}$ $(1.60 \mathrm{~cm})$, which were significant over each other. The genotype effect and interaction between treatment and genotype was insignificant in terms of micro-shoot length. Under our experimental condition, low and moderate callusing was observed when the nodal segments treated with 2.0 and $3.0 \mathrm{mg} / \mathrm{l}$ BAP along with $0.05 \mathrm{mg} / \mathrm{l} \mathrm{NAA}$ respectively. It is well known that cytokinins are essential 
for axillary bud formation, growth and shoot multiplication. Among the cytokinins reported BAP is widely used in tissue culture as it is synthetic and stable in nature. Earlier, Kumar et al., (2003) and Gupta et al., (2013) reported the use of $2.0 \mathrm{mg} / \mathrm{l} \mathrm{BAP}$ alone or in a combination of $0.5 \mathrm{mg} / \mathrm{l} \mathrm{NAA}$ for better culture establishment of different marigold varieties and apetalous male sterile lines. Majumder et al., (2014) reported that a lower concentration of BAP $(1.0 \mathrm{mg} / \mathrm{l})$ along with $\mathrm{GA}_{3}$ was found to be most effective in marigold cv. Pusa Narangi Gainda in culture establishment. They also reported the formation of callus along with micro-shoot when NAA added along with BAP and $\mathrm{GA}_{3}$. These conflicting results could be attributed to the use of different species as well as the possible effects of different genotypes. Our previous experiment with Seracole varieties also showed that the higher concentrations of BAP along with NAA lead to callus formation resulted in the poor establishment of cultures in marigold (Ravindra et al., 2017). It is evident from Table 3 that maximum establishment index (130.2) was obtained in MS medium supplemented with $2.0 \mathrm{mg} / \mathrm{l} \mathrm{BAP}$ $+0.05 \mathrm{mg} / \mathrm{l} \mathrm{NAA}$, which was statistically significant over all other treatments. Establishment index was very low (28.80) in MS medium devoid of hormones (control). Among the genotypes, maximum establishment index was reported in PBG (70.63) followed by PA (68.88). However, establishment index was statistically insignificant among the two genotypes. Interaction between growth regulator and genotype show non-significant differences among each other.

\section{Effect of different growth regulators on shoot proliferation}

It is clearly evident from the Table 4 that, maximum $(100 \%)$ proliferation of microshoots was recorded on MS medium supplemented with devoid of any hormones (control), $0.5 \mathrm{mg} / \mathrm{l} \mathrm{BAP}+0.1 \mathrm{mg} / \mathrm{l} \mathrm{NAA}, 0.5$ $\mathrm{mg} / \mathrm{l}$ Kinetin $+0.1 \mathrm{mg} / \mathrm{l} \mathrm{NAA}$ and $0.5 \mathrm{mg} / \mathrm{l}$ $\mathrm{BAP}+0.1 \mathrm{mg} / \mathrm{l} \mathrm{NAA}+2.5 \mathrm{mg} / \mathrm{AgNO} \mathrm{AgN}_{3}$. Under our experimental conditions, both the genotypes were unable to establish and proliferate where the concentrations of BAP and Kinetin more than $0.5 \mathrm{mg} / \mathrm{l}$. Vitrified shoots and profuse callusing was frequently observed in higher cytokinin concentrations which lead to poor establishment of cultures in proliferation media (Fig $3 \mathrm{c}$ ). It is also evident from the data (Table 4) that significantly higher proliferation with healthy micro-shoots and dark green leaves were observed when the media was supplemented with $0.5 \mathrm{mg} / \mathrm{l} \mathrm{BAP}+2.5 \mathrm{mg} / 1 \mathrm{AgNO}_{3}+0.1$ $\mathrm{mg} / \mathrm{l}$ NAA compared to all other growth regulators (Fig $3 \mathrm{a} \& b$ ). Senescence of leaves, shoot tip death and longer intermodal lengths were observed in MS media supplemented with $0.5 \mathrm{mg} / \mathrm{l} \mathrm{BAP}+0.1 \mathrm{mg} / \mathrm{l} \mathrm{NAA}$ devoid of $\mathrm{AgNO}_{3}$. In general longest shoots $(4.1 \mathrm{~cm})$ were observed in control followed by MS medium supplemented with $0.5 \mathrm{mg} / \mathrm{l} \mathrm{BAP}+$ $2.5 \mathrm{mg} / \mathrm{AgNO}_{3}+0.1 \mathrm{mg} / \mathrm{l} \mathrm{NAA}$. But, the rate of proliferation was very low in control over $0.5 \mathrm{mg} / \mathrm{l}$ BAP supplemented media (T10). No significant differences were recorded in between two genotypes and also in genotype and treatment interaction.

Among the different treatments, maximum (4.3, 18.8, 64.2 and 208.2 shoots/explant) shoots were obtained on MS medium supplemented with BAP $(0.5 \mathrm{mg} / \mathrm{l})+\mathrm{NAA}$ $(0.1 \mathrm{mg} / \mathrm{l})+2.5 \mathrm{mg} / \mathrm{l} \mathrm{AgNO}_{3}$ which was statistically significant with BAP $(0.5 \mathrm{mg} / \mathrm{l})+$ NAA $(0.1 \mathrm{mg} / \mathrm{l})(3.0,8.7,26.7$ and 82.3 shoots/ explant) in 30, 60, 90 and 120 days respectively. The two way interaction between growth regulator and genotype was found to be significant in 30, 60, 90 and 120 days. The interaction between treatment and genotype revealed that maximum (237.7) shoots were obtained from Pusa Basanti 
Gainda which was significantly differed with Pusa Arpita (178.7 shoots) when they cultured on MS medium supplemented with BAP (0.5 $\mathrm{mg} / \mathrm{l})+\mathrm{NAA}(0.1 \mathrm{mg} / \mathrm{l})+\mathrm{AgNO}_{3}(2.5 \mathrm{mg} / \mathrm{l})$ after 120 days. These results indicate the maximum proliferation in African marigold cv. PBG over French marigold cv. PA (Fig 4).

Misra and Datta (1999) reported that addition of kinetin, 2, 4-D and higher levels of BAP in proliferation media of white marigold culture produced undesirable callus, hyperhydrated leaves and vitrified shoots. $\mathrm{GA}_{3}$ causes browning of shoot tips in proliferation media. Misra and Datta (2001) also reported the use of low concentration of BAP $(1.1 \mathrm{mM})$ along with $\mathrm{AgNO}_{3}$ (29.41 mM) for better shoot proliferation. Silver nitrate is known to promote multiple shoot formation in different plants. In vitro shoot formation was improved by incorporating silver nitrate in the culture medium (Kumar et al., 2009). The present findings lend support from the previous work done by Misra and Datta (1999, 2000 and 2001). These results are also tantamount with our earlier results with Seracole marigold genotypes (Ravindra et al., 2017).

From the present studies, it is concluded that by using the standardized protocols, ornamentally high valued marigold lines can be taken up for the production of true-to-type, disease free quality planting material in large scale. This can also be helpful for long term maintenance of African and French marigold germplasm, valuable breeding lines and other biotechnological related works.

\section{References}

Anonymous, 2015. Indian Horticulture Database, Available from, URL: http://nhb.gov.in/areapro/horst_galance_2016.pdf

Gupta, Y. C., Vaidya, P., Dhiman, S. R., and Sharma, P. 2013. In vitro propagation and maintenance of genetic male sterility in marigold. Progressive Horticulture. 45 (1): 152-159.

Hojnik, M., Skerget, M., and Knez, Z. 2008. Extraction of lutein from Marigold flower petals - Experimental kinetics and modelling. LWT-Food Science and Technology. 41(10): 2008-2016.

Kumar, A., Raghava, S. P. S., Singh, S. K., and Misra, R. L. 2003. Micropropagation of male sterile marigold plants for $F_{1}$ hybrid seed production. Indian Journal of Ornamental Horticulture. 6(1): 1-6.

Kumar, V., Parvatam, G., and Ravishankar, G. A. 2009. $\mathrm{AgNO}_{3}-$ a potential regulator of ethylene activity and plant growth modulator. Electronic Journal of Biotechnology. 12(2): 1-15.

Majumder, J., Singh, K. P., Singh, S. K., Prasad, K. V., and Verma, M. 2014. In vitro morphogenesis in marigold using shoot tip as explant. Indian Journal of Horticulture 71(1): 82-86.

Misra, P., and Datta, S. K. 1999. In vitro proliferation of white marigold (Tagetes erecta L.) through shoot tip proliferation. Current Science. 77(9): 1138-1140.

Misra, P., and Datta, S. K. 2000. In vitro maintenance of $\mathrm{F}_{1}$ hybrid. Current Science. 78(4): 383-385.

Misra, P., and Datta, S. K. 2001. Direct differentiation of shoot buds in leaf segments of white marigold (Tagetes erecta L.). In vitro cellular and developmental biology. 37: 466-470.

Murashige, T., and Skoog, F. 1962. A revised medium for rapid growth and bioassays with tobacco tissue cultures. Physiologia Plantarum. 15: 473-497.

Nazki, Imtiyaz, T., Siddique, M. A. A., Rather, Z. A., Mir, M. A., and Bhat, M. A. 2015. An improvised low cost hardening protocol for in vitro raised plantlets of Gerbera jamesonii. Indian 
Journal of Agriculture Science. 85(1): 43-46.

Qi, Y., Ye, Y., and Bao, M. 2011. Establishment of plant regeneration system from another culture of Tagetes patula. African journal of Biotechnology. 10(75): 17332-17338.

Ravindra Kumar, K., Singh, K. P., Raju, D. V. S., Panwar, S., Bhatia, R., Jain, P. K., and Vinod. 2017. Standardization of rapid multiplication protocol in petaloid male sterile lines of African marigold (Tagetes erecta) through in vitro culture. Indian Journal of Agricultural Sciences. 87 (10): 1295-1302.

Sekioka, T. A., and Tanaka, J. S. 1981. Differentiation in callus culture of cucumber (Cucumis sativus L). Hort Science. 16:451.

Sen, M. K., Hassan, M. M., Nasrin, S., Jamal, M. A. H. M., Rashid, A. N. M. M., and Dash, B. K. 2013. In vitro sterilization protocol for micro propagation of Achryranthes aspera L. node. International Research Journal of Biotechnology. 4(5): 89 -93.

Singh, N. V., Singh, S. K., and Patel, V. B. 2011. In vitro culture establishment studies on pomegranate. Indian Journal of Horticulture. 68(3): 307-311.

Tejaswini, Sane, A., Gadre, A., and Ghatke, M. 2016. Characterisation and utilization of three distinct male sterile systems in marigold (Tagetes erecta). Indian Journal of Agriculture Sciences. 86(10): 1271-1275.

Verma, A. K., Prasad, K. V., Janakiram, T., and Kumar, S. 2012. Standardization of protocol for pre-treatment, surface sterilization, regeneration, elongation and acclimatization of Chrysanthemum morifolium Ramat. International Journal of Horticulture. 2(3): 7-12.

\section{How to cite this article:}

Ravindra Kumar, K., Kanwar Pal Singh, D.V.S. Raju, Sapna Panwar, Reeta Bhatia, Surendra Kumar and Pavanesh Kumar Verma. 2018. Standardization of in vitro Culture Establishment and Proliferation of Micro-Shoots in African and French Marigold Genotypes. Int.J.Curr.Microbiol.App.Sci. 7(01): 2768-2781. doi: https://doi.org/10.20546/ijcmas.2018.701.332 